\title{
Experimental study on mechanical properties of steel - sand interface
}

\author{
Zhaoxiao Fang ${ }^{1, *}$, Weijiang Wang ${ }^{2}$, and Zhaoli Fang ${ }^{3}$ \\ ${ }^{1}$ Key Laboratory of Civil Engineering Disaster Prevention and Mitigation, Shandong University of Science and Technology, Qingdao \\ 266590, China; \\ ${ }^{2}$ College of electronic and information engineering, Shandong University of Science and Technology, Qingdao 266590, China; \\ ${ }^{3}$ College of chemistry and chemical engineering, Jangxi Science and Technology Normal University, Nanchang 330013, China
}

\begin{abstract}
The interface between steel and sand can be regard as a steel-sand system, and its mechanical properties have an important role in many geotechnical applications. The mechanical properties of various steel-sand interfaces classified by sand mean particle size $D_{50}$ were investigated through interface shear tests. The results show that for a given steel-sand interface, the peak strength of the interface increase with increasing axial pressure. As the $D_{50}$ value increases, the cohesions for steel-sand interfaces decrease, while the friction angles of the interfaces first increase and then decrease. In the process of shearing, the shrinkage of steel-sand interface occurs, mainly due to the broken of sand particles. The decrease in interface friction coefficients due to an increase in axial pressure was observed. Particle size distribution has a significant effect on the interface friction coefficient of steel-sand interface. When the average particle size $D_{50}$ changes from $0.1 \mathrm{~mm}$ to $0.47 \mathrm{~mm}$, the friction coefficient of steel-sand interface increases by $134 \%-161 \%$.
\end{abstract}

\section{Introduction}

The testing of soil-structure interfaces plays an important role in the analysis of geotechnical applications such as pile foundations, retaining walls, and earth reinforcement ${ }^{[1]}$. These applications rely on the mechanical properties of the interfaces between different soils (sand, clay, and gravel) and structural materials (steel, concrete, wood, and geosynthetic). Based on laboratory researches, the mechanical properties of these interfaces is largely dependent on loading conditions, soil properties, and structural material types ${ }^{[2-3]}$.

Goodhue et al. ${ }^{[4]}$ found that the friction angles of geosynthetic-dense sand interface generally ranges between $39^{\circ}$ and $43^{\circ}$, and the cohesions ranges between 17 and $28 \mathrm{kPa}$. The effect of sand density is weak for the friction angle of steel-sand interface ${ }^{[5]}$. Lings et al. ${ }^{[6]}$ concluded that the peak friction coefficient decrease with the increase of normal stress when the dilation of the steel-sand interface occurs. A large number of experimental studies have been carried out to investigate the mechanical properties of the interfaces between different soils and structural materials over the years. However, the effects of sand particle size gradation on steel-sand interface mechanical properties have rarely been investigated.

In this paper, the mechanical properties of steel-sand interfaces were studied by interface shear tests. The effects of sand particle size gradation on the interface mechanical properties were investigated by analyzing the relationships between mean particle size $D_{50}$ and steel-sand interface parameters (cohesion, friction angle, and friction coefficient). The relationship between axial displacement and horizontal displacement was obtained, and it can be found that the shrinkage of steel-sand interface occurs in the process of shearing, mainly due to the broken of sand particles.

\section{Test process}

\subsection{Interface shear apparatus}

A series of interface shear tests on the mechanical properties of steel-sand interface could be carried out using the GDS interface shear apparatus (Figure 1). Furthermore, the shear area could remain constant during tests. The torque, the axial pressure, and the shear rate could be increased linearly or nonlinearly. The $360^{\circ}$ rotating shear platform (Figure 2) could be capable of achieving large enough shear angle displacements. And the large deformation analysis for steel-sand interface could be conducted. Therefore, the GDS interface shear apparatus has great advantages in analyzing the mechanical properties of steel-sand interface.

\subsection{Experimental materials}

The sands used from the Yellow Sea of Qingdao were classified by the fine, medium, and coarse sands based on mean particle size $D_{50}$. The physical properties of the

* Corresponding author: 1964288403@qq.com 
sands were measured. Such parameters are summarized in Table 1. Particle size distribution of the sands is shown in Figure 3.

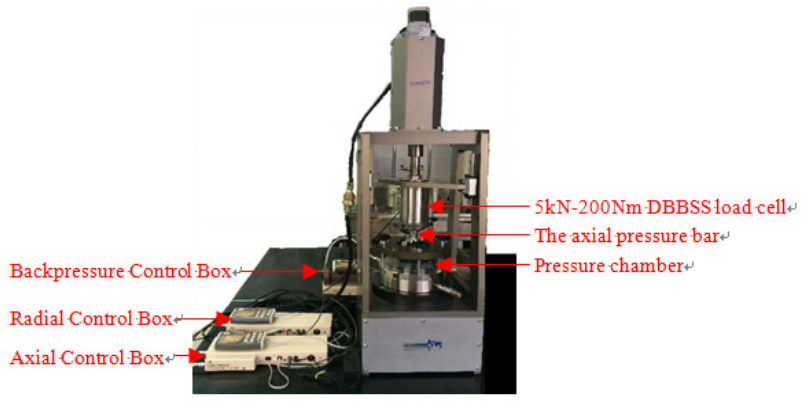

Fig. 1. GDS interface shear apparatus

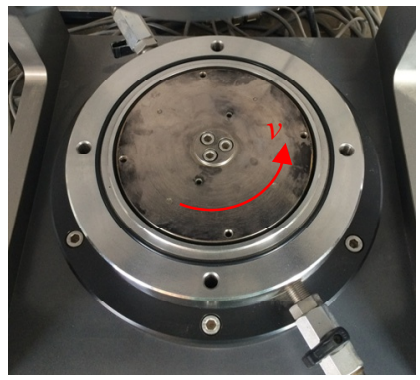

Fig. 2. The shear platform

Table 1. Physical properties of the sands used

\begin{tabular}{|c|c|c|c|c|c|}
\hline Testing sands & $e_{\min }$ & $e_{\max }$ & $D_{50} / \mathrm{mm}$ & $C_{\mathrm{u}}$ & $C_{\mathrm{c}}$ \\
\hline fine sand & 0.61 & 0.90 & 0.10 & 1.78 & 1.00 \\
\hline medium sand & 0.72 & 1.03 & 0.47 & 1.86 & 0.92 \\
\hline coarse sand & 0.72 & 1.00 & 0.74 & 1.50 & 0.97 \\
\hline
\end{tabular}

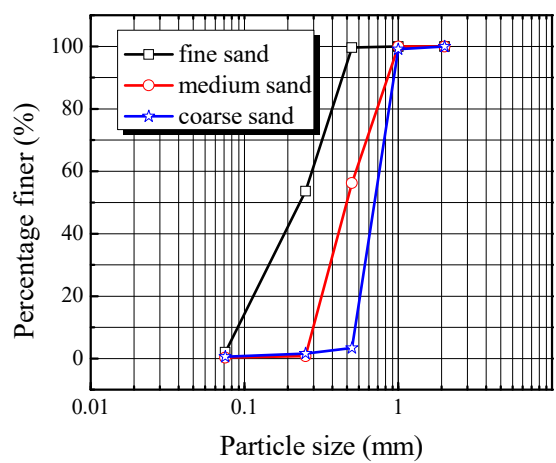

Fig. 3. Particle size distribution of the sands

\subsection{Test scheme}

The interfacial shear test data were used to determine the effects of sand particle size gradation on the mechanical properties of steel-sand interfaces. It is to be noted that there are three types of steel-sand interfaces (i.e., steel-fine sand interface (SFSI), steel-medium sand interface (SMSI), steel- coarse sand interface (SCSI)). The initial relative density of samples is taken as 0.9 (dense). Firstly, specimens are consolidated one-dimensionally to the designated axial pressures (100,
$150,200,250,300,350$, and $400 \mathrm{kPa})$. Then, interface shear tests are conducted with a speed of $1.0 \mathrm{~cm} / \mathrm{min}$ until a total shear displacement of $8 \mathrm{~mm}$ is reached.

The steel-sand interface consists of the steel plate surface which is in contact with sand in addition to a thin layer of adjacent sand, as shown in Figure 4.

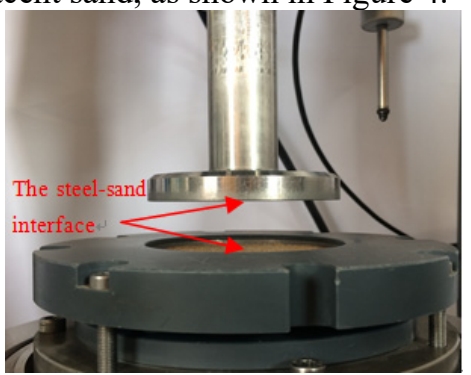

Fig. 4. The steel-sand interface

\section{Results and discussion}

\subsection{Effects of sand particle size gradation on shear strength}

Figure 5 shows some typical curves of shear stress versus horizontal displacement for the various interfaces, where the shear stress has a general tendency to increase rapidly with the horizontal displacement increasing. And finally the shear stress remains a stable value after reaching the peak strength. For a given axial pressure, the coarser the sand particle is, the larger the shear strength of the interface is. This is because the surface roughness and movement form of sand particles with different particle sizes are different, resulting in different shear characteristics of the steel-sand interface. It should be noted that the shear strength of SMSI is equal to that of SCSI under axial pressure of 100 and $300 \mathrm{kPa}$.

Based on the Mohr-Coulomb strength theory, failure envelopes for the steel-sand interfaces(SSI) are shown in Figure 6. Each point represents the peak strength of the interface under a certain axial pressure. Moreover, there is an approximately linear correlation between the shear strength and axial pressure. It is also found that sand particle size gradation has a marked influence on the peak strength of the various interfaces (except SCSI). When the axial pressure is $400 \mathrm{kPa}$, the peak strength for SCSI of $463.78 \mathrm{kPa}$ is increased by about $55 \%$ compared with that of SFSI, $299.43 \mathrm{kPa}$.

The relationships between shear strength parameters and mean particle size $D_{50}$ are showed in Figure 7, indicating that sand particle size gradation has a strong influence on the shear strength parameters of the various interfaces. The cohesions are calculated to be 19.91, 4.32, and $1.52 \mathrm{kPa}$ for SFSI, SMSI, SCSI, respectively. And the friction angles are calculated to be $34.44^{\circ}, 49.02^{\circ}$, and $48.98^{\circ}$, respectively. As can be seen from Figure 7, with the increase of $D_{50}$ value, the cohesions of SSI decrease, but with the increase of $D_{50}$ value, the friction angles of the interfaces initially increase and then decrease. 


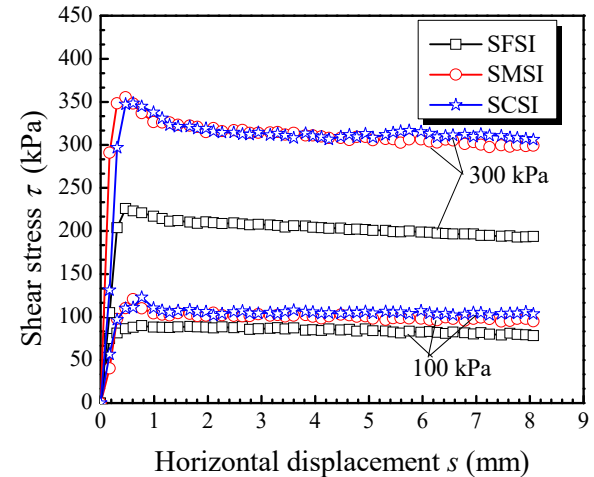

Fig. 5. Variations of shear stress versus horizontal displacement for the steel-sand interfaces

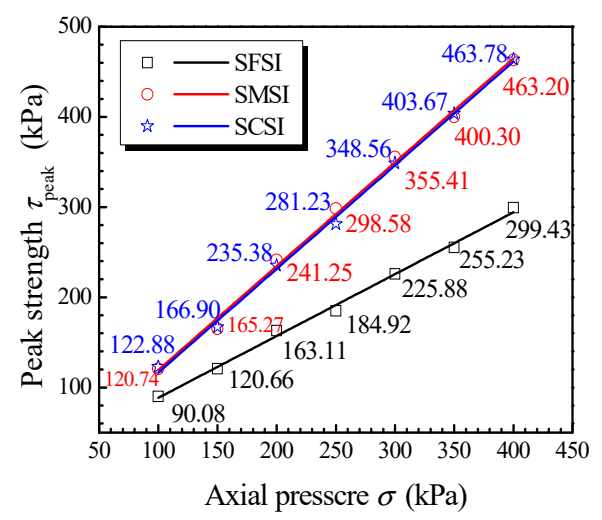

Fig. 6. The Mohr-Coulomb failure envelopes

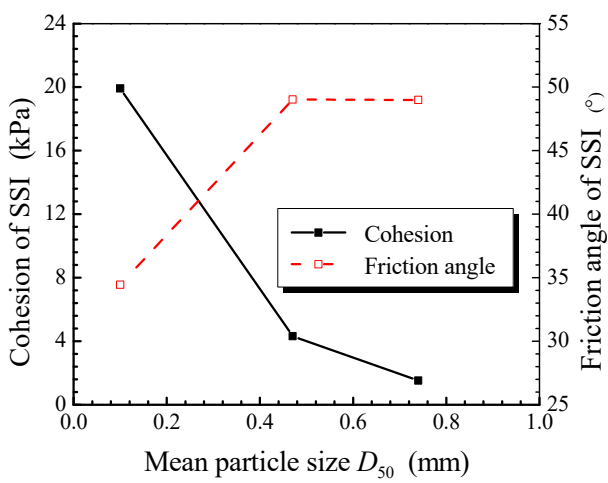

Fig. 7. The cohesion and friction angle for the steel-sand interfaces

\subsection{The relationship between axial displacement and horizontal displacement}

As the shear surface remains constant, the volumetric change of sample is reflected in the axial displacement of the shear surface. When the axial displacement is negative, the specimen decreases in volume on account of the shrinkage for the steel-sand interface. With the axial displacement of positive, the volume of sample expands due to the dilation of the steel-sand interface under a certain axial pressure.

The results are presented in a plot of axial displacement versus horizontal displacement as shown in Figure 8. It can be found that the axial displacements of the various interfaces increase with an increase in the horizontal displacement, indicating that the shrinkage of the steel-sand interfaces occurs in the process of shearing. The reason for this shrinkage behavior can be understood by referring to Figure 9, where the changes of the sand surface before and after shearing are shown. Before shearing (Figure 9(a)), the cutting ring is filled with coarser sand particles. And there are many voids between the sand particles. In Figure 9(b), the coarser sand particles adjacent to the steel surface are broken into a large number of finer sand particles. Due to the movement of those particles, they will fill up the voids of the lower part for sample. Meanwhile, the height for the sample of $H_{0}$ (before shearing) is reduced by $\delta_{1}$ compared with that of $H_{1}$. It also can be seen from Figure 9(c) that the increased amount of the axial displacement is equal to $\delta_{2}$ due to the rearrangement of finer sand particles. The numbers of voids in the sample and finer sand particles produced increase with an increase in sand particle size, as well as the $\delta_{1}$ and $\delta_{2}$ values. Therefore, the shrinkage of the steel-sand interfaces occurs in the process of shearing, mainly due to the broken of sand particles. And there are SCSI, SMSI, and SFSI, being in the descending order of the final axial displacements. In addition, the axial displacements for the various interfaces are basically identical with the horizontal displacements of less than $0.3 \mathrm{~mm}$. When the horizontal displacements are less than $1.0 \mathrm{~mm}$, the corresponding axial displacements are approximately 3-4 times larger than those of the horizontal displacements within the range of 1.0-8.0 mm, indicating that the value of $\delta_{1} / \delta_{2}$ is in the range of 3-4. With the axial pressure of $300 \mathrm{kPa}$, the final axial displacement for SMSI, $0.09 \mathrm{~mm}$, is increased by 0.03 $\mathrm{mm}$ compared with that of SFSI, $0.06 \mathrm{~mm}$.

Combining Figure 6 and 8, it can be found that the peak strength of the steel-sand interface can be reached in the process of the broken of coarser sand particles, suggesting that the broken of sand particles has a remarkable influence on the interface peak strength. With the rearrangement of finer sand particles, the interface shear stress gradually reaches the residual strength. Horizontal displacement $s(\mathrm{~mm})$

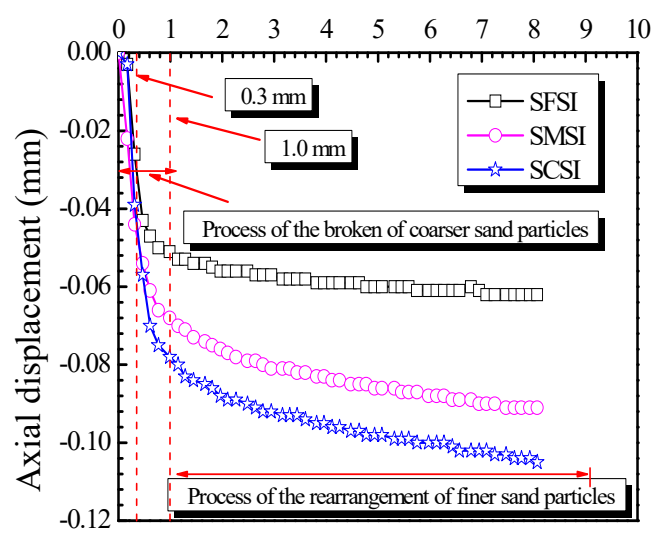

Fig. 8. Variations of axial displacement versus horizontal displacement under axial pressure of $300 \mathrm{kPa}$ 


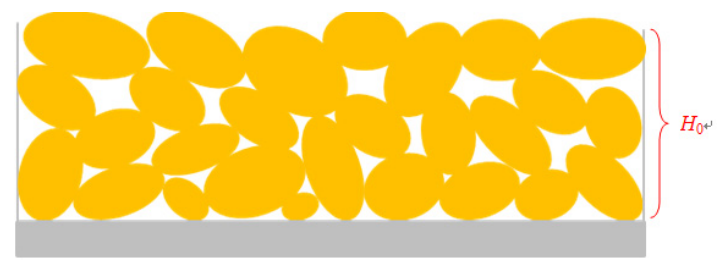

(a) The sand surface before shearing

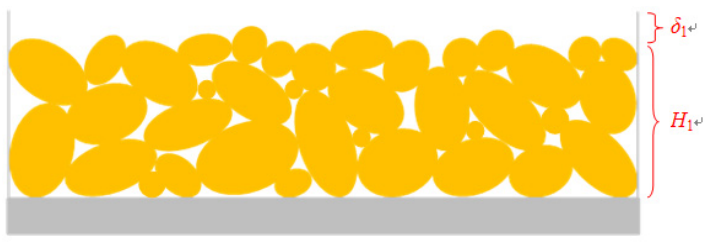

(b) Process of the broken of coarser sand particles

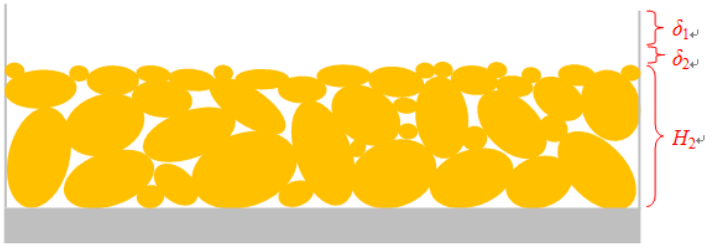

(c) Process of the rearrangement of finer sand particles

Fig. 9. Changes for the sand surface before and after shearing

\subsection{Effects of sand particle size gradation on interface friction coefficient}

Besides friction angle and cohesion, the friction coefficient $f_{\mathrm{s}}$ is introduced to analyze the differences of the mechanical properties for various steel-sand interfaces, can be written as:

$$
f_{\mathrm{s}}=\frac{\tau_{\text {peak }}}{\sigma}
$$

where $\tau_{\text {peak }}$ is the peak strength of steel-sand interface, $\sigma$ is the axial pressure.

Figure 10 illustrates the relationship between friction coefficient and axial pressure. Figure 10 presents that, when the axial pressure exceeds $250 \mathrm{kPa}$, the effects of sand particle size gradation on interface friction coefficient are weak for SSI, concluding that the friction coefficients for SFSI, SMSI, and SCSI slightly fluctuate with increasing axial pressure.

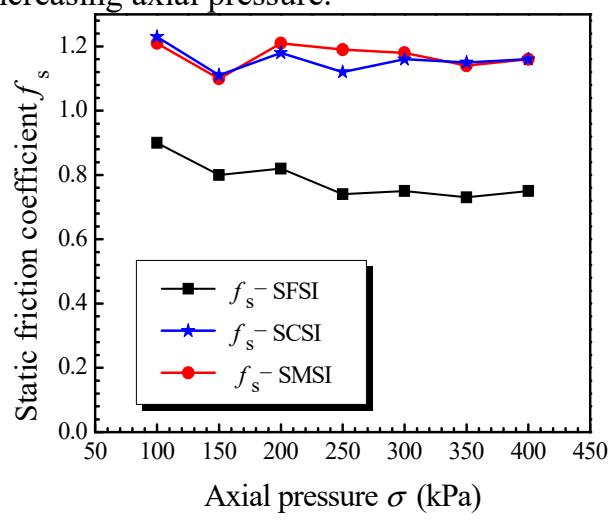

Fig. 10. Relationship between friction coefficient and axial pressure
The relationship between friction coefficient and mean particle size $D_{50}$ is shown in Figure 11, suggesting that sand particle size gradation has a marked influence on the interface friction coefficients for SSI. Figure 11 presents that the friction coefficients for SSI first rapidly increase and then slowly increase (or decrease under axial pressure of 200 and $250 \mathrm{kPa}$ ) with the increase of mean particle size $D_{50}$.

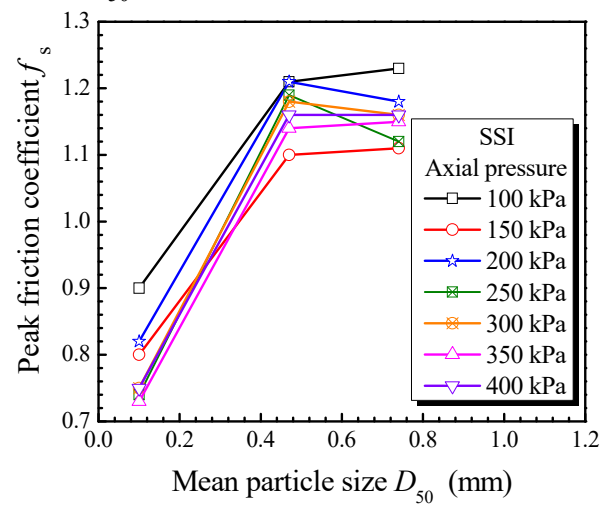

Fig. 11. Relationship between friction coefficient and mean particle size $D_{50}$

\section{Conclusions}

(1) For a given steel-sand interface, the shear strength for the interface increases with an increase in axial pressure. With the increase of the $D_{50}$ value, there is a decrease in the cohesions for SSI. However, the friction angles of the interfaces first increase and then decrease with increasing the $D_{50}$ value.

(2) The shrinkage of steel-sand interface occurs in the process of shearing, mainly due to the broken of sand particles. When the horizontal displacements are less than $1.0 \mathrm{~mm}$, the corresponding axial displacements are approximately 3-4 times larger than those of the horizontal displacements within the range of 1.0-8.0 $\mathrm{mm}$.

(3) When the axial pressure exceeds $250 \mathrm{kPa}$, the friction coefficients for SFSI, SMSI, and SCSI slightly fluctuate with the axial pressure increasing.

\section{References}

1. Mortara G, Mangiola A, Ghionna V N. Canadian Geotechnical Journal, 44, 739-752(2007).

2. Zhang G, Zhang J M. Progress in Natural Science: Materials International, 19, 1187-1196(2009).

3. Saberi M, Annan C D, Konrad J M. International Journal of Non-Linear Mechanics, 102, 1-13(2018).

4. Goodhue M J, Edil T B, Benson C H. Journal of Geotechnical and Geoenvironmental Engineering, 2001, 127, 353-362(2017).

5. Tiwari B, Ajmera B, Kaya G. Geotechnical Special Publ, 71,1747-1756(2010).

6. Lings M L, Dietz M S. Soils and Foundations , 45,1-14(2008). 